\title{
The Gaia-ESO Survey: Lithium depletion in the Gamma Velorum cluster and inflated radii in low-mass pre-main-sequence stars
}

\author{
R. D. Jeffries ${ }^{1 \star}$, R. J. Jackson ${ }^{1}$, E. Franciosini ${ }^{2}$, S. Randich ${ }^{2}$, D. Barrado ${ }^{3}$, A. Frasca ${ }^{4}$, \\ A. Klutsch ${ }^{4}$, A. C. Lanzafame ${ }^{4,5}$, L. Prisinzano ${ }^{6}$, G. G. Sacco $^{2}$, G. Gilmore ${ }^{7}$, A. Vallenari ${ }^{8}$, \\ E. J. Alfaro ${ }^{9}$, S. E. Koposov ${ }^{7,10}$, E. Pancino ${ }^{2,11}$, A. Bayo ${ }^{12}$, A. R. Casey ${ }^{7}$, M. T. Costado ${ }^{9}$, \\ F. Damiani ${ }^{6}$, A. Hourihane ${ }^{7}$, J. Lewis ${ }^{7}$, P. Jofre ${ }^{7,13}$, L. Magrini ${ }^{2}$, L. Monaco ${ }^{14}$, L. Morbidelli ${ }^{2}$, \\ C. C. Worley ${ }^{7}$, S. Zaggia ${ }^{8}$ and T. Zwitter ${ }^{15}$ \\ ${ }^{1}$ Astrophysics Group, Keele University, Keele, Staffordshire ST5 5BG, United Kingdom \\ 2 INAF - Osservatorio Astrofisico di Arcetri, Largo E. Fermi 5, 50125, Florence, Italy \\ ${ }^{3}$ Depto. Astrofisica, Centro de Astrobiologia (INTA-CSIC), ESAC campus, Camino Bajo del Castillo s/n, E-28692 Villanueva de la Cañada, Spain \\ ${ }^{4}$ INAF - Osservatorio Astrofisico di Catania, via S. Sofia 78, 95123, Catania, Italy \\ ${ }^{5}$ Dipartimento di Fisica e Astronomia, Sezione Astrofisica, Universitá di Catania, via S. Sofia 78, 95123, Catania, Italy \\ ${ }^{6}$ INAF - Osservatorio Astronomico di Palermo, Piazza del Parlamento 1, 90134, Palermo, Italy \\ ${ }^{7}$ Institute of Astronomy, University of Cambridge, Madingley Road, Cambridge CB3 OHA, United Kingdom \\ ${ }^{8}$ INAF - Padova Observatory, Vicolo dell'Osservatorio 5, 35122 Padova, Italy \\ ${ }^{9}$ Instituto de Astrofisica de Andalucía-CSIC, Apdo. 3004, 18080, Granada, Spain \\ 10 Moscow MV Lomonosov State University, Sternberg Astronomical Institute, Moscow 119992, Russia \\ ${ }^{11}$ ASI Science Data Center, Via del Politecnico SNC, 00133 Roma, Italy \\ 12 Instituto de Física y Astronomía, Universidad de Valparaiíso, Chile \\ ${ }^{13}$ Núcleo de Astronomía, Facultad de Ingeniería, Universidad Diego Portales, Av. Ejercito 441, Santiago, Chile \\ ${ }^{14}$ Departamento de Ciencias Fisicas, Universidad Andres Bello, Republica 220, Santiago, Chile \\ ${ }^{15}$ Faculty of Mathematics and Physics, University of Ljubljana, Jadranska 19, 1000, Ljubljana, Slovenia
}

\begin{abstract}
We show that non-magnetic models for the evolution of pre-main-sequence (PMS) stars cannot simultaneously describe the colour-magnitude diagram (CMD) and the pattern of lithium depletion seen in the cluster of young, low-mass stars surrounding $\gamma^{2}$ Velorum. The age of $7.5 \pm 1 \mathrm{Myr}$ inferred from the CMD is much younger than that implied by the strong Li depletion seen in the cluster M-dwarfs and the Li depletion occurs at much redder colours than predicted. The epoch at which a star of a given mass depletes its Li and the surface temperature of that star are both dependent on its radius. We demonstrate that if the low-mass stars have radii $\sim 10$ per cent larger at a given mass and age, then both the CMD and Li depletion pattern of the Gamma Vel cluster are explained at a common age of $\simeq 18-21$ Myr. This radius inflation could be produced by some combination of magnetic suppression of convection and extensive cool starspots. Models that incorporate radius inflation suggest that PMS stars similar to those in the Gamma Vel cluster, in the range $0.2<M / M_{\odot}<0.7$, are at least a factor of two older and $\sim 7$ per cent cooler than previously thought and that their masses are much larger (by $>30$ per cent) than inferred from conventional, non-magnetic models in the Hertzsprung-Russell diagram. Systematic changes of this size may be of great importance in understanding the evolution of young stars, disc lifetimes and the formation of planetary systems.
\end{abstract}

Key words: stars: magnetic fields; stars: low-mass - stars: evolution - stars: pre-mainsequence - clusters and associations: general - starspots

\section{INTRODUCTION}

Precise measurements of main-sequence K- and M-dwarf radii in eclipsing binaries have revealed alarming discrepancies between theoretical models and observations. For a given mass, the abso- 
lute radii of stars with $0.2<M / M_{\odot}<0.8$ can be $\sim 10$ per cent larger than predicted and hence, for a given luminosity, the effective temperature is overestimated by $\sim 5$ per cent (e.g. LopezMorales \& Ribas 2005; Morales et al. 2009a,b; Torres 2013). Since interferometric radii for nearby, slowly-rotating low-mass stars are much closer to "standard", non-magnetic evolutionary models (e.g. Demory et al. 2009; Boyajian et al. 2012), it is possible that the radius inflation of fast-rotating binary components is explained by dynamo-generated magnetic activity (Morales, Ribas \& Jordi 2008). The exact mechanism is debated, but could be magnetic fields inhibiting convection throughout the star (Mullan \& MacDonald 2001; Feiden \& Chaboyer 2013, 2014) or cool, magnetic starspots that block outward flux at the stellar surface (MacDonald \& Mullan 2013; Jackson \& Jeffries 2014b).

If magnetic activity is implicated in inflating radii, then the same effect should also be present in low-mass pre main sequence (PMS) and zero-age main sequence (ZAMS) stars, which are frequently found to be as fast-rotating and magnetically active as the tidally-locked components of older eclipsing binary systems. Besides revealing an interesting new facet of the astrophysics of lowmass stars, radius inflation in PMS stars would have important practical consequences. Models that include such inflation lead to substantial increases in both the ages and masses that would be inferred from colour-magnitude diagrams (CMDs) and Hertzsprung-Russell diagrams (HRDs; perhaps by factors of two or more - see Somers \& Pinsonneault 2015b; Feiden 2016), systematically affecting studies of the star formation process and the early evolution of stars and their planetary systems by altering the inferred timescales of important phases (Bell et al. 2013; Soderblom et al. 2014).

At present it is not possible to directly measure the masses, radii and ages of young low-mass stars in order to compare them with evolutionary models. However, it has been suggested that discrepancies between masses, radii, temperatures and luminosities in PMS binaries (Kraus et al. 2015. 2016), the lithium depletion dispersion in PMS stars (Somers \& Pinsonneault 2015a,b) and the anomalous colours of PMS and ZAMS stars with respect to model isochrones (Stauffer et al. 2003; Covey et al. 2016 and see Section 5 ), might be explained by radius inflation or magnetic activity. Indirect evidence for enlarged stellar radii in young clusters, based on the product of their rotation periods and projected equatorial velocities, supports this view (Jackson, Jeffries \& Maxted 2009; Jackson et al. 2016).

The Gaia-ESO spectroscopic survey of representative stellar populations at the ESO Very Large Telescope (GES, Gilmore et al. 2012; Randich \& Gilmore 2013) includes observations of large, unbiased samples of PMS stars in young clusters, hence providing an important dataset to further investigate these issues. This paper presents the results of a test, first suggested by Yee \& Jensen (2010, see also Feiden 2016 and Messina et al. 2016), that exploits the sensitive mass and radius dependence of the onset of lithium depletion in fully convective PMS stars. Using GES observations of lithium, we find strong evidence for radius inflation in a large group of lowmass PMS stars belonging to the Gamma Vel cluster (Jeffries et al. 2009). We show that standard, non-magnetic models fail to simultaneously describe the CMD and the pattern of lithium depletion, but that a simple inflation of the radius at a given mass and age could solve this problem.

\section{THE GAMMA VEL CLUSTER AND NON-MAGNETIC MODELS}

The Gamma Vel cluster is a group of several hundred, low-mass PMS stars and some tens of higher mass ZAMS stars, that are spatially concentrated around the massive Wolf-Rayet binary system $\gamma^{2}$ Vel (Pozzo et al. 2000; Jeffries et al. 2009). The cluster was one of the first targets for the GES; intermediate-resolution ( $R \simeq 17000$ ) fibre spectroscopy in the cluster, taken with the Giraffe spectrograph and HR15N grating ( $\lambda \lambda$ 6444-6816 $)$, has been extensively described and analysed in Jeffries et al. (2014) and Prisinzano et al. (2016), establishing a kinematically coherent cluster of low-mass PMS stars at an age of $\sim 10 \mathrm{Myr}$. The cluster appears to have two kinematic components, separated by just $2 \mathrm{~km} \mathrm{~s}^{-1}$, that are almost indistinguishable in terms of their positions in CMDs and the lithium depletion seen among their low-mass members. No distinction is made between these subgroups in this paper.

The intrinsic distance modulus and reddening to the Gamma Vel cluster have been determined as $7.76 \pm 0.07 \mathrm{mag}$ and $E(B-$ $V)=0.038 \pm 0.012 \mathrm{mag}$, from main-sequence fitting to the higher mass population (Jeffries et al. 2009). This distance is consistent with the wider Vela OB2 association, of which the Gamma Vel cluster appears to be a part, and also with interferometric distances to the $\gamma^{2}$ Vel binary, which however appears to be younger than the associated PMS stars. The metallicity of the cluster was found to be $[\mathrm{Fe} / \mathrm{H}]=-0.057 \pm 0.018$ from GES high resolution spectra of its solar-type stars (Spina et al. 2014).

The GES spectra were used by Jeffries et al. (2014) and Prisinzano et al. (2016) to confirm membership for $>200$ low-mass PMS stars with $12<V<19$, corresponding approximately to $1.5>M / M_{\odot}>0.2$ for an assumed age of $10 \mathrm{Myr}$. The catalogue and photometry of confirmed and probable members published as table 5 in Prisinzano et al. (2016) is used here. The sample was restricted to those stars with radial velocities between 14 and $22 \mathrm{~km} \mathrm{~s}^{-1}$ and a reliable measurement of (or upper limit to) the equivalent width of the $\mathrm{Li}$ I $6708 \AA$ line (EWLi), in order to reduce the possibility of contamination to just a few out of the remaining 198 stars. The absolute $V$ magnitude vs $(V-I)_{0}$ (Cousins photometry) CMD for these stars is shown in Fig. 1a, corrected for the distance modulus, an extinction $A_{V}=0.13$ and reddening $E(V-I)=0.055$ (assumed from scaling $E(B-V)$ ). Superimposed on the data are solar-composition isochrones taken from the Baraffe et al. (2015, hereafter BHAC15) and the Dotter et al. (2008, hereafter Dartmouth) models, The Dartmouth models were transformed into colour-magnitude space with the relationships between bolometric corrections and $T_{\text {eff }}$ implied by the BHAC 15 models.

The new BHAC15 models, based on a revision of the BTSettl atmospheres and molecular linelists described by Allard (2014), provides a much better description of the optical CMDs of low-mass stars than previous evolutionary models. Fig. 1a shows isochrones at 7.5, 14 and $30 \mathrm{Myr}$; there is almost no difference between the BHAC15 and Dartmouth isochrones and both provide an excellent match across the entire data range at an age of $\simeq 7.5 \mathrm{Myr}$, estimated by minimising the median offset in $V$ between data and isochrones for the lowest $2 / 3$ of the data points. ${ }^{1}$ The small uncertainties in distance, reddening and placement of the isochrone contribute to an age precision of just $\sim \pm 1 \mathrm{Myr}$.

1 About one third (e.g. Fischer \& Marcy 1992) of the points in the CMD will be unresolved multiple systems with high enough mass ratios to cause them to be brighter and perhaps redder than their single siblings. Filtering these out minimises their influence on the inferred age. 

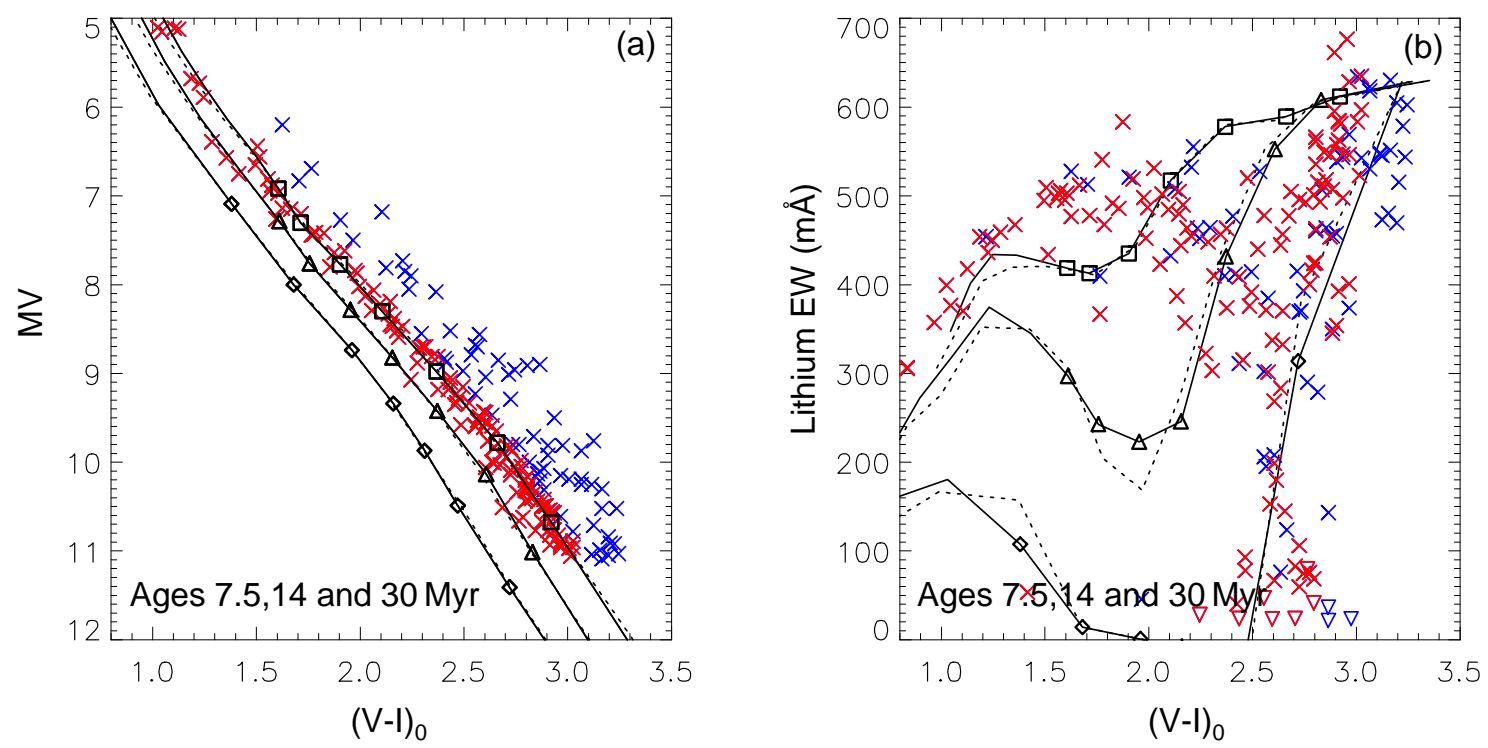

Figure 1. The absolute magnitude versus intrinsic colour diagram and lithium depletion pattern in the Gamma Vel cluster. The left hand panel shows the absolute $V$ magnitude versus intrinsic $(V-I)_{0}$ CMD for a set of members defined in Prisinzano et al. (2016). Overplotted are isochrones from the Baraffe et al. (2015, solid) and Dotter et al. (2008, dashed) isochrones at 7.5 (diamonds), 14 (triangles) and 30 Myr (squares). A distance modulus of 7.76 mag, $A_{V}=0.13$ mag and $E(V-I)=0.055$ mag have been assumed. Mass points from $0.2 M_{\odot}$ to $0.8 M_{\odot}$ in $0.1 M_{\odot}$ steps are indicated by the open symbols on each isochrone. The right hand panel shows Li $6708 \AA$ equivalent width versus colour for the same cluster members, with predicted isochrones from the same set of models and at the same ages as the left hand panel. The blue points in each plot mark possible unresolved binaries, chosen as the third of points furthest away from the best-fitting isochrone in the CMD. The median uncertainty in EWLi is $15 \mathrm{~m} \AA$. Downward pointing triangles indicate upper limits.

Figure $1 \mathrm{~b}$ shows the same set of stars in a plot of equivalent width of EWLi vs the $V-I$ colour. The pattern of Li abundance and therefore EWLi as a function of temperature or equivalently, colour, is a sensitive function of mass and age in young low-mass stars. In contracting, fully convective PMS stars, photospheric depletion commences when the centre reaches Li-burning temperatures $\left(\geqslant 3 \times 10^{6} \mathrm{~K}\right)$ and convection rapidly mixes Li-depleted material to the surface (e.g. Bildsten et al. 1997). The virial theorem tells us that Li-burning starts when the mass-to-radius ratio increases to a critical value (Jackson \& Jeffries 2014a); models with solar metallicity suggest that in a coeval group of stars, Li-burning should commence first in $\simeq 0.5 M_{\odot}$ stars at $\simeq 5 \mathrm{Myr}$, extend to a narrow range of higher and lower mass stars over the next $20 \mathrm{Myr}$, followed by complete depletion for stars $<0.6 M_{\odot}$ by $120 \mathrm{Myr}$ (e.g. see Fig. 2b).

In Gamma Vel the observational data show a rise from a small EWLi in the hotter stars towards a plateau at $V-I \simeq 2$, but with a clear Li depletion "dip" centered at $V-I=2.7 \pm 0.2$. There $\sim 20$ stars at the bottom of this dip that have EWLi $<100 \mathrm{~m} \AA$, and which must have depleted their lithium by more than 3 orders of magnitude according to curves of growth for EWLi (see below). Superimposed on the data we show Li depletion isochrones predicted by the same models shown in Fig. 1a. The Li depletion factors predicted by these models have been converted from Li abundances into EWLi assuming an initial $\mathrm{Li}$ abundance of $A\left(\mathrm{Li}_{0}\right)=3.3$ and by folding the predicted $A(\mathrm{Li}), T_{\text {eff }}$ coordinates at each mass and age through LTE curves of growth for the Li I $6708 \AA$ feature, defined by Soderblom et al. (1993) for $T_{\text {eff }}>4200 \mathrm{~K}$ and by Zapatero-Osorio et al. (2002) for $T_{\text {eff }}<4200 \mathrm{~K}^{2}{ }^{2}$ The colours are from the models as for Fig. $1 \mathrm{~b}$ and reddening has been applied.

2 The predicted EWLi for a given $\mathrm{Li}$ abundance and $T_{\text {eff }}$ from the
Both sets of models (BHAC15 and Dartmouth) are quite similar and cannot fit the Li depletion data at all. At the age of 7.5 Myr implied by the CMD there is far too little Li depletion (by orders of magnitude in $\mathrm{Li}$ abundance and factors of several in EWLi). An age of $>14 \mathrm{Myr}$ is needed to provide the required Li depletion, but this would still occur at colours that are far too blue by $>0.5$ mag. Only a small part of this age discrepancy could be explained by unresolved binarity making some of the Gamma Vel stars redder - the most likely candidate binaries, those well above the 7.5 Myr isochrone in Fig. 1a, are indicated. In Appendix A (see Fig. A1 a,b) it is shown that exactly the same result is arrived at from the $V$ vs $V-K$ CMD and EWLi vs $V-K$ diagram. Neither can the additional Li depletion be explained by an anomalous metallicity. Although Li depletion is metallicity sensitive, in the sense that greater depletion is expected in more metal-rich stars of a given mass, Spina et al. (2014) have established that the metallicity of the Gamma Vel cluster is slightly subsolar (see the Introduction).

In summary, the most commonly used, contemporary lowmass stellar evolution models are unable to simultaneously describe the CMD and Li depletion pattern of PMS stars in the Gamma Vel cluster.

\section{MODELS WITH INFLATED RADII}

If stars of a given mass and age have larger radii and cooler $T_{\text {eff }}$ than predicted by the standard models, then their central temperatures will be lower and their Li depletion will be delayed; however that depletion will occur in stars with redder colours. This, along

Zapatero-Osorio et al. estimates are within 10 per cent of those independently calculated by Palla et al. (2005) at $T_{\text {eff }} \leqslant 3600 \mathrm{~K}$. 
with the context described in the Introduction, leads to consideration of whether radius inflation could solve the problem identified in Section 2.

The BHAC15 models predict that stars with $M<0.7 M_{\odot}$, deplete their lithium whilst descending near-vertical, fullyconvective Hayashi tracks in the HRD; the luminosity of the star results principally from the release of gravitational energy. In this case a simple polytropic model may be used to investigate the effects of reduced heat transfer in the surface layers of a star on the evolution of stellar radius, luminosity and $\mathrm{Li}$ abundance. As discussed in the Introduction, a number of authors have experimented with evolutionary models that incorporate the effects of rapid rotation and magnetic fields, either in the form of inhibited convection throughout the star, or the blocking of flux at the surface by cool starspots. It is well known that low-mass PMS stars (including those in Gamma Vel) do rotate rapidly and show high levels of magnetic activity (Frasca et al. 2015). Here, we remain agnostic about which, if any, of these mechanisms might be effective in lowmass PMS stars, but hypothesise that something causes them to be larger at a given mass and age by reducing the radiative flux at the surface.

Jackson and Jeffries (2014a) considered the effect of starspots reducing the luminosity of PMS stars by a factor $(1-\beta)$ relative to the luminosity of an unspotted star at the temperature of the unspotted photosphere. Equations 2 and 3 of that paper show that the effect of starspots is to reduce the luminosity, $L$ whilst increasing the radius, $R$ of a star relative to an unspotted star of similar mass $M$ and age $t$ such that:

$\left[\frac{R}{R_{0}}\right]_{M} \propto(1-\beta)^{-D} t^{-D}$

$\left[\frac{L}{L_{0}}\right]_{M} \propto(1-\beta)^{D} t^{D-1}$,

where $D=(A-4) /(3 A-4)$ and $A=\partial \log L / \partial \log T_{\text {eff }}$ at fixed mass. For near-vertical Hayashi tracks $A$ is very large and the exponent $D \simeq 1 / 3$, i.e. the radius at a given age increases with spot coverage as $(1-\beta)^{-1 / 3}$, while the luminosity decreases in equal proportion. It follows that, for a fixed mass, the age at which the central temperature $(\propto M / R)$ reaches the ignition point of lithium (see Bildsten et al. 1997) is increased by a factor $(1-\beta)^{-1}$.

Whilst this model of reduced heat transfer at the stellar surface was used to investigate the effect of starspots it can equally be used to model other effects that inflate stars on Hayashi tracks at a given $M$ and $t$. Specifically, it can approximately represent the effects of reducing the efficiency of convective heat transfer due to interior magnetic fields or some other reduction in the convective mixing length provided that the majority of the star remains adiabatic. This is expected to be true for young PMS stars, since super-adiabatic layers are limited to quite close to the stellar surface (see fig. 10 in Feiden \& Chaboyer 2014). In this case $\beta$ is simply related to the relative increase in radius, $R / R_{0}$ at fixed $M$ and $t$ as $\beta=$ $1-\left(R / R_{0}\right)^{-3}$, i.e. a 10 per cent increase in radius corresponds to $\beta=0.25$.

In Fig. 2a we show how this polytropic model modifies the BHAC15 isochrones and the positions of stars of a given mass in the HRD for $\beta=0.25$. The inflated models are calculated for masses $0.2<M / M_{\odot}<0.8$ where the stars are (almost) fully convective at the ages considered here. Stars of a given mass and age become slightly less luminous and significantly cooler. As a result, any age inferred from matching these models to data in the CMD would be significantly older and a mass estimated from po- sition in the HRD would be larger than estimated from standard models.

In Fig. $2 \mathrm{~b}$ we show how the same polytropic model affects isochrones in the $\mathrm{Li}$ abundance- $T_{\text {eff }}$ plane. These are calculated by assuming that the same relationship between $M / R$ (and hence central temperature) and Li depletion holds for both standard and inflated models. Inflation increases the radius at a given mass and age and hence decreases the central temperature. A star of a given mass takes longer to reach the Li-burning stage, but has a cooler $T_{\text {eff }}$ when it does so. The result is less Li depletion at a given age, but the centre of the "Li-dip" becomes cooler by $\sim 300 \mathrm{~K}$.

\section{COMPARISON OF MODELS WITH INFLATED RADII TO THE GAMMA VEL CLUSTER}

Whilst the relationship between $\beta$, radius inflation and $\mathrm{Li}$ depletion is to first order independent of whether the inflation is caused by starspots or something else, the consequent appearance of the star is not. The effective temperature is reduced by a factor $(1-\beta)^{1 / 4}$. For a star with uniform surface temperature, the effect on $V$ and $V-I$ is calculated straightforwardly using the relationships between luminosity, temperature, absolute magnitudes and colours implied by the BHAC15 models (ignoring the small gravity change). For spotted stars the light comes from both spotted and unspotted regions. The magnitudes and colours will depend on $\beta$ and the ratio of the temperatures of the spotted and immaculate photosphere. The procedure for calculating this is described in Appendix B.

In Figs. 3a,b inflated (by 10 per cent at a given mass and age) versions of isochrones from the BHAC15 and Dartmouth models are compared with the Gamma Vel cluster data. The distance modulus and reddening are as described in Section 2. Li abundances are converted to EWLi using the same curves of growth described in Section 2. Figure 3a shows that for 10 per cent inflation, an 18-21 Myr isochrone is now an excellent match to the CMD using either the inflated BHAC15 or inflated Dartmouth models. This is significantly older than found in Section 2 because stars of a given mass and age are cooler in the inflated models. Figure $3 \mathrm{~b}$ shows 18 and $21 \mathrm{Myr}$ Li depletion isochrones for these inflated models, illustrating how extremely age-dependent the Li depletion is at this epoch. $\mathrm{A} \sim 20 \mathrm{Myr}$ isochrone is now a much better fit, both to the depth and location of the Li-dip in the Gamma Vel data, than was seen in Fig. 1b. The inflated BHAC15 and Dartmouth models are similar in terms of predicted Li-dip depth, but the inflated Dartmouth models fare slightly worse in that the colour of their predicted Li-dip is still slightly blueward of the observations. There is no a priori reason to suppose that one $\beta$ is appropriate for stars at all masses, but a uniform value does seem to work reasonably well.

Uncertainties on the required values of $\beta$ and consequent ages were estimated in the following way. The required age was determined from the EWLi-colour plot. An initial $\beta$ value was chosen, which defined a narrow range of isochrones that provided a minimum in EWLi somewhere between $300 \mathrm{~m} \AA$ and undetectable. The centre of this age range in turn defines a new $\beta$ value (and hence level of radius inflation) that will provide a match to the CMD using the same procedure used to define the CMD age in Section 2. This procedure is then iterated using the new $\beta$ value and converges on the parameters and age ranges shown in Table 1 . The colour of the Li-dip is not used as a fitting criterion. The finite precision of the age estimate inferred from the non-magnetic models $(7.5 \pm 1 \mathrm{Myr})$ formally gives an uncertainty of \pm 0.04 in the final $\beta$ estimates, but 

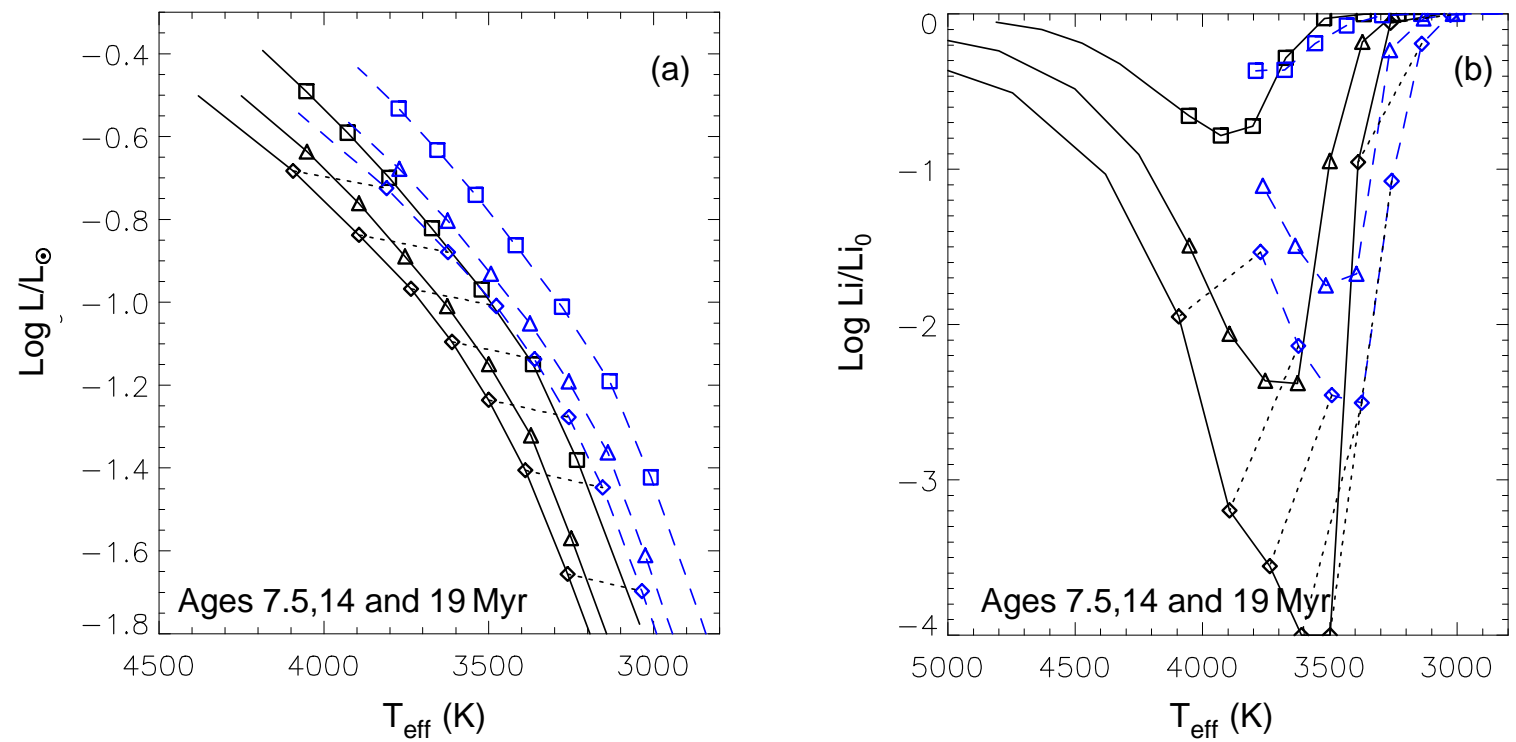

Figure 2. The effects of a 10 per cent radius inflation $(\beta=0.25)$. The left hand panel shows isochrones in the Hertzsprung-Russell diagram from the BHAC15 model at 7.5 (diamonds), 14 (triangles) and $19 \mathrm{Myr}$ (squares). The blue dashed lines show the same isochrones, modified for the effects of radius inflation. Mass points from $0.2 M_{\odot}$ to $0.8 M_{\odot}$ in $0.1 M_{\odot}$ steps are indicated by the open symbolds on each isochrone. A set of black dotted lines indicate (for the 19 Myr isochrone) how the effects of inflation modify each mass point. The inflated 19 Myr isochrone lies very close to the 7.5 Myr BHAC15 isochrone. The right hand panel shows the same isochrones in the Li abundance versus $T_{\text {eff }}$ plane. These diagrams illustrate that for a given mass and age, the effect of radius inflation is to reduce the luminosity, lower the surface temperature and reduce the amount of Li depletion.

Table 1. Best fitting ages for inflated models

\begin{tabular}{|c|c|c|c|c|c|}
\hline $\begin{array}{l}\text { Type of } \\
\text { model }\end{array}$ & $\beta^{a}$ & $\begin{array}{l}\text { Radius } \\
\text { inflation }^{b}\end{array}$ & $\begin{array}{c}\text { Temperature } \\
\text { ratio }^{c}\end{array}$ & $\begin{array}{c}\text { Spot } \\
\text { coverage }^{d}\end{array}$ & $\begin{array}{l}\text { Age } \\
\text { (Myr) }\end{array}$ \\
\hline Uniform & 0.25 & $10 \%$ & 0.93 & - & $18-21$ \\
\hline Spots & 0.30 & $13 \%$ & 0.90 & 0.87 & $19-22$ \\
\hline Spots & 0.41 & $19 \%$ & 0.85 & 0.86 & $23-26$ \\
\hline Spots & 0.51 & $27 \%$ & 0.80 & 0.86 & $28-32$ \\
\hline
\end{tabular}

$a$ The fraction of flux blocked at the surface.

$b$ The level of inflation compared to non-magnetic models.

$c$ For uniform models this is the ratio of $T_{\text {eff }}$ to the $T_{\text {eff }}$ of an uninflated star of the same mass. For the spotted models this is the ratio of spotted to unspotted temperatures $T_{s p} / T_{s}-$ see Appendix B.

$d$ The surface coverage fraction of cooler spots.

leads to only an additional $1 \mathrm{Myr}$ uncertainty in the age (which is ignored).

Figures 3a,b also show equivalent isochrones at 18 and $21 \mathrm{Myr}$ predicted by the "magnetic Dartmouth models" described in Feiden \& Chaboyer (2014) and Feiden (2016). These implement magnetic inhibition of convection constrained by a boundary condition of an average $2.5 \mathrm{kG}$ equipartition field at the stellar surface. At a given mass, and an age of $\sim 20 \mathrm{Myr}$, these models predict that stars are inflated by between 12 per cent at $0.8 M_{\odot}$ and 7 per cent at $0.2 M_{\odot}$. These models are quite close to our simple polytropic models in the CMD, but are too blue at the lowest masses. In the EWLi versus colour diagram, the depth and colour of the Li-dip are similar to our simple models in Fig. 3b, perhaps providing a worse fit to the Gamma Vel data on the red (low-mass) side of the Li-dip, but a better fit on the blue side. The former discrepancy is likely due to the lower level of inflation at lower masses than assumed in our best fitting uniform temperature and $\beta$ polytropic models. The latter is because at $\sim 20 \mathrm{Myr}$, the magnetic Dartmouth models have a small radiative core at $M \geqslant 0.6 M_{\odot}$, meaning that the base of the convection zone is cooler than the core; this reduces the amount of photospheric Li depletion over our simple fully convective polytropic approximation, better matching the data.

The models shown in Figs. 3a,b assume that the inflated stars have a uniform surface temperature, but the polytropic model can equally describe inflation due to dark starspots (see Section 2). We find that the reddening of the isochrones is maximised for the case of a uniform temperature; spotted models produce a smaller redward shift for the same $\beta$ and level of inflation. This means that if the radius inflation is caused by spots then agreement between the CMD age and lithium depletion ages requires a larger $\beta$, more radius inflation and older ages than for a uniform temperature reduction. Consequently, spotted models require a very large spot coverage if they are to be the sole cause of the radius inflation. Results for uniform temperature reductions and spotted models are summarised in Table 1, and Figs. 3c,d show examples of inflated models that match the CMD and Li depletion pattern for an assumed spot temperature ratio of $T_{s p} / T_{s}=0.85$ (see Appendix B).

\section{DISCUSSION}

In Section 2 we showed that the most commonly used evolutionary models cannot simultaneously describe the CMD and Li depletion pattern of the Gamma Vel cluster at a common age. Ages based on $\mathrm{Li}$ appear to be much older, though we note that these models do not describe Li depletion at any age, because they predict Li depletion occuring at much bluer colours than observed. Based on what is known about the radii of magnetically active stars in eclipsing lowmass binaries, it is natural to seek a resolution in terms of modifying the mass-radius relationship at low-masses. Figures 3 and A1 

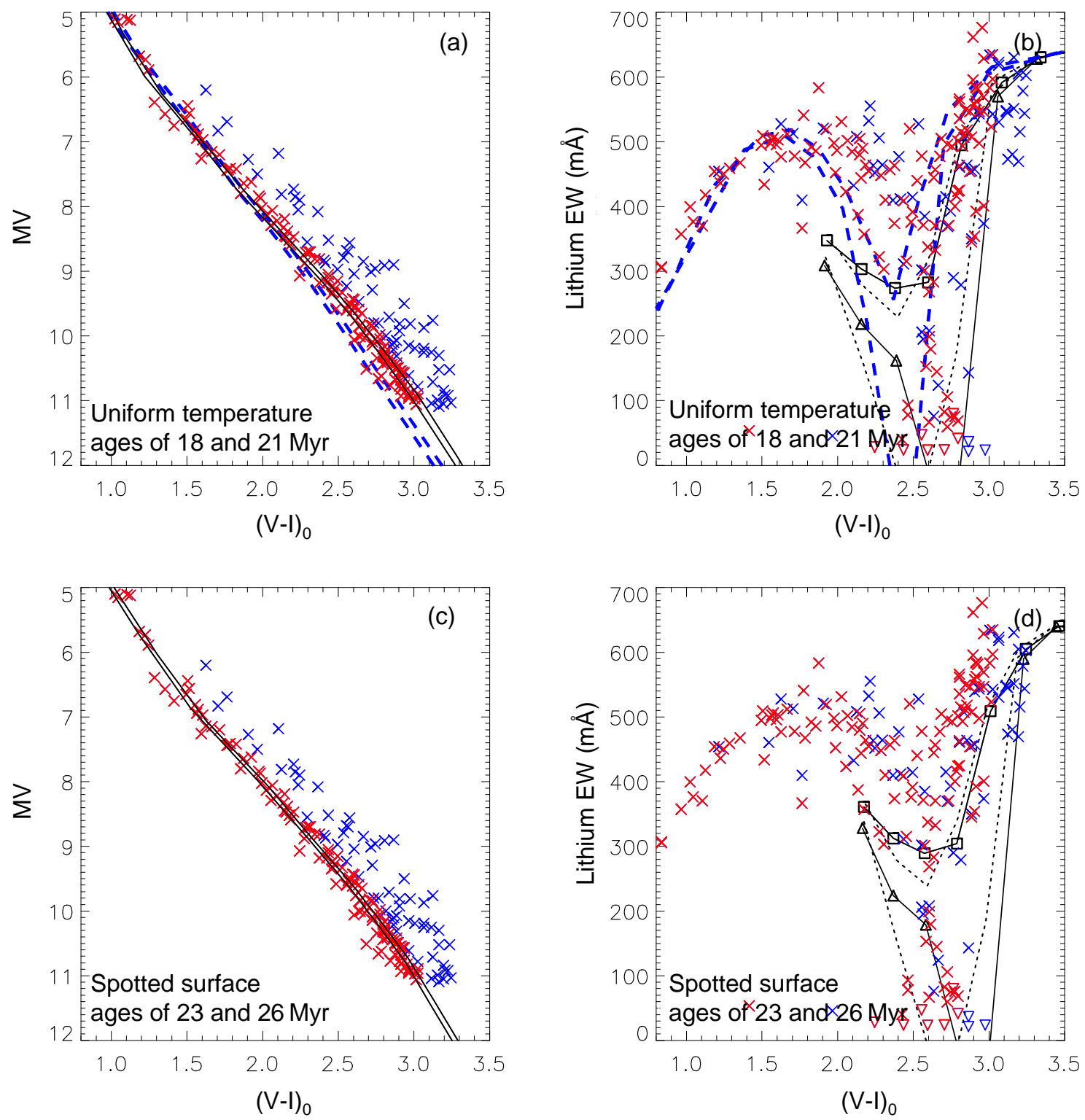

Figure 3. The absolute magnitude versus intrinsic colour diagram and lithium depletion pattern in the Gamma Vel cluster (same data and symbol colour meaning as for Fig. 1) compared with isochrones at 18 and 21 Myr modified by radius inflation, with mass points from $0.2 M_{\odot}$ to $0.8 M_{\odot}$ indicated by open symbols. Panels (a) and (b) refer to the uniform temperature radius inflation with $\beta=0.25$, while panels (c) and (d) are similar diagrams for a spotted model with $\beta=0.41$ and a spotted to unspotted temperature ratio of 0.85 . Black solid lines and black short-dashed lines (almost coincident with the black solid lines in panels (a) and (c) and hence not visible) are BHAC15 and Dartmouth isochrones modified by inflation according to the polytropic model described in Section 3 at the ages indicated in the plots. In panels (a) and (b) the blue dashed lines shows 18 and 21 Myr isochrones from the magnetic Dartmouth models.

show that a resolution is possible if the radii of stars are inflated with respect to standard models by about 10 per cent at a given mass and age. The results of a simple polytropic model are in remarkably good agreement with the "magnetic" Dartmouth models of Feiden \& Chaboyer (2014) for $M \leqslant 0.6 M_{\odot}$, which show similar levels of inflation and are also a reasonable match to the data. Starspots could also be responsible for radius inflation. Our modelling (see Table 1) suggests that if they were to be the sole cause of the discrepancy between HRD/CMD ages and Li-depletion ages, then this would require significantly more radius inflation and an unrealistically high level of spot coverage - spot modulation and doppler imaging can give only lower limits to spot coverage, but modelling of TiO bands in the spectra of magnetically active stars suggets 50 per cent coverage may be possible (e.g. O'Neal et al. 2004 and extensive discussion in Jackson \& Jeffries 2014b). In reality, these active stellar photospheres are more likely to feature a distribution of temperatures rather than the simple uniform or bimodal extremes assumed by our models and the radius inflation would be due to a combination of magnetically moderated effects.

Yee \& Jensen (2010, see also Malo et al. 2014) noted a quantitatively similar discrepancy between ages from the HRD and ages inferred from Li depletion for stars in the young Beta Pic moving group. They also suggested that radius inflation might be a way to resolve the difference, noting that inflation would increase ages 
from the HRD and would shift Li depletion to cooler $T_{\text {eff }}$. However, they suggested that inferred ages from $\mathrm{Li}$ depletion would be reduced by radius inflation. In fact as we have shown here, and has also been demonstrated by Jackson \& Jeffries (2014a) and Somers \& Pinsonneault $(2014,2015 a)$, radius inflation delays the onset of Li depletion leading to older inferred ages. As a result, the ages from the CMD/HRD and Li-depletion can only be reconciled at ages at least a factor of two older than inferred from the CMD/HRD using standard models. This assumes that the inflationary factors were already in place prior to commencement of $\mathrm{Li}$ burning. If magnetic activity is the culprit then this seems reasonable, since high levels of magnetic activity are ubiquitously seen in much younger PMS stars than those considered here.

Almost identical conclusions were reached for the Beta Pic group by Messina et al. (2016). They showed that a version of the magnetic Dartmouth models was able to describe the CMD and Li-depletion patterns at a common age of $25 \pm 3 \mathrm{Myr}$. This is entirely consistent with our analysis and age of 18-21 Myr for the Gamma Vel cluster using similar models - an empirical comparison of the Li depletion patterns in the two datasets (compare Fig. A1 with fig. 1 from Messina et al.) shows that Gamma Vel is definitely younger than Beta Pic; the lithium-dip is much wider in Beta Pic (by about 0.5 mag in $V-K$ ), and extends to significantly bluer colours. However, given the extreme age sensitivity of Li depletion, an age difference of a few Myr is all that is required.

Radius inflation due to magnetic activity has the capacity to explain a number of observational puzzles that have emerged in recent years concerning young stars. The increase in age that would follow from using models with radius inflation solves a long-standing discrepancy between the ages derived from the HRD/CMD of low-mass PMS stars using standard models and the considerably older ages determined using the ZAMS turn-on or nuclear turn-off ages from higher mass stars with little magnetic activity (e.g. Naylor 2009; Pecaut et al. 2012). Bell et al. (2013) found that by adopting empirical relationships between bolometric correction and colour derived using a fiducial sequence of (magnetically active) ZAMS stars in the Pleiades cluster, they could bring the ages derived from high- and low-mass stars in young clusters into agreement. The empirical modification to the bolometric corrections is equivalent to changing the radius of the low-mass stars at a given $T_{\text {eff }}$. More recently, Feiden (2016) showed that the magnetic Dartmouth models (discussed in Section 3) inflated the radii and increased the derived ages of low-mass PMS stars in the Upper Sco association by a factor of two, bringing them into agreement with ages determined from higher mass stars.

The large age increases suggested here apply to young clusters for which the age has been previously determined using isochronal fits to their low-mass PMS stars. Ages derived from the "lithium depletion boundary" (LDB) - the luminosity below which Li remains unburned in the low-mass stars of clusters with ages from 20-130 Myr (e.g. Bildsten et al. 1997), will be considerably less affected. LDB ages have been proposed as the most model-independent of age determination methods in young clusters (Soderblom et al. 2014) and indeed Jackson \& Jeffries (2014a) showed that these ages are only increased by $\sim(1-\beta)^{-0.5}$ due to magnetically inflated radii. Thus a 10 per cent radius inflation requiring $\beta=0.25$ would increase the determined LDB age by just 15 per cent. For example Binks \& Jeffries (2016) showed that adopting the inflated Dartmouth magnetic models discussed here increased the LDB age of the Beta Pic moving group from $21 \mathrm{Myr}$ to $24 \mathrm{Myr}$.

There are also discrepancies between model predictions and the measured radii and masses for low-mass PMS and ZAMS eclipsing binary systems in clusters and associations (Kraus et al. 2015, 2016; David et al. 2016; Sandberg Lacy et al. 2016). On the basis of position in the HRD, evolutionary models predict masses that are too low and ages that are much younger than suggested by higher mass stars in the same clusters/systems. Similarly, the dynamical masses of astrometric PMS binaries are higher than implied by their measured luminosities and standard evolutionary models (Hillenbrand \& White 2004; Azulay et al. 2015; Rizzuto et al. 2016). Figure 2a demonstrates that these phenomena would be expected if stars were inflated by $\sim 10$ per cent with respect to the standard evolutionary models $-T_{\text {eff }}$ is reduced by 7 per cent, inferred ages are increased by a factor of $\geqslant 2$, whilst masses inferred from the HRD/CMD are almost doubled at the lowest masses considered here (from $0.2 M_{\odot}$ to $\sim 0.35 M_{\odot}$ ), with a smaller effect at higher masses (from $0.6 M_{\odot}$ to $\sim 0.8 M_{\odot}$ ). If masses were estimated from luminosities (or absolute magnitudes), the effect would be much smaller.

Finally, an explanation of the well known issue of a rotationdependent dispersion of $\mathrm{Li}$ abundance at a given $T_{\text {eff }}$ in ZAMS stars (e.g. Soderblom et al. 1993; Barrado et al. 2016) has been proposed by Somers \& Pinsonneault (2014, 2015a,b), which assumes that stars have radii that are inflated by differing amounts depending on their level of rotation and magnetic activity. This leads to differing levels of Li depletion, as explained in Section 3, and a dispersion in $\mathrm{Li}$ abundances at the ZAMS that was imprinted during PMS evolution. This dispersion is important because it illustrates the need for a solution that can vary from star-to-star, rather than a global change to evolutionary models that would affect all stars of the same mass equally. For instance, similar radius inflation could be produced by artificially reducing the convective efficiency or altering the adopted relationship between temperature and optical depth in the atmosphere (Chen et al. 2014), but this could not explain any dispersion in stellar properties.

A considerable dispersion in EWLi at $V-I \sim 2.7 \pm 0.2$ is present in the Gamma Vel data and cannot be explained by uncertainties in EWLi alone ${ }^{3}$. The presence of stars with undetectable levels of $\mathrm{Li}$ can also not be explained in terms of contamination by non-members; there are too many with a radial velocity close to the cluster centroid and all have some secondary indication of a youthful nature - see Prisinzano et al. (2016) for details. If this dispersion were present at a given $T_{\text {eff }}$ or colour, it would favour an explanation in terms of differences in $\beta$ caused by differing magnetic activity levels or rotation rates from star-to-star. Here, the simplified assumption has been made of a single value of $\beta$ across the mass range, which could perhaps be justified in terms of the consistent (saturated) levels of chromospheric activity exhibited by PMS stars in Gamma Vel despite a spread in rotation rates (see Frasca et al. 2015). On the other hand, it is uncertain how closely the levels of spot coverage or interior magnetic field strengths are correlated with chromospheric activity - a Li-depletion-rotation connection has recently been identified in the similar PMS stars of NGC 2264 (age 5-10 Myr) by Bouvier et al. (2016). Attempts to investigate this in Gamma Vel are hampered by a lack of rotation period data and by both intrinsic and measurement uncertainties. Photometric precision, variability, small reddening differences, differences in the spot coverage when the photometry was performed, the presence of unresolved binary companions and the possibility of a small

3 The median uncertainty in EWLi is $15 \mathrm{~mA}$, see fig. 5 in Prisinzano et al. (2016) 
age spread in the population (see Jeffries et al. 2014) can all contribute to a scatter in $V-I$ and EWLi that could obscure any relationship between Li depletion and rotation/activity over such a narrow Li-dip. The nature of the EWLi dispersion in Gamma Vel and in other young clusters observed by GES is deferred to another paper.

A remaining puzzle is the status of the massive binary $\gamma^{2}$ Vel, which appears to be at the centre of the cluster. According to calculations by Eldridge (2009), the binary has an age of $5.5 \pm 1 \mathrm{Myr}$. This age discrepancy between the $\gamma^{2}$ Vel and the PMS star surrounding it was already noted by Jeffries et al. $(2009,2014)$. The introduction of inflated models for the PMS stars makes the discrepancy larger. Data from the Gaia satellite should ultimately settle whether $\gamma^{2}$ Vel and the lower mass stars are physically related or are merely a line of sight coincidence.

\section{SUMMARY}

The colour magnitude diagram of low-mass PMS stars in the Gamma Vel cluster is well-fitted with isochrones from the most commonly used evolutionary models at an age of $7.5 \pm 1$ Myr. However, the same models cannot explain the pattern of Li depletion in the same stars at the same age - there is too little Li depletion and it occurs at colours that are too blue. A simple polytropic model that simulates the effects of starspots or magnetic fields in inhibiting the radiative flux at the surface of a fully convective star is used to modify the evolutionary models, showing that stars become larger, less luminous and cooler at the same mass and age. These revised models are able to simultaneously fit the CMD and the Li depletion pattern - at a significantly increased age of 18-21 Myr if radii are inflated by 10 per cent and the surface temperatures are uniform, or at even older ages if the radii are solely inflated due to a very large covering fraction of cool starspots. These conclusions are very similar to those arrived at by Messina et al. (2016) who performed a combined analysis of the CMD and Li depletion pattern for stars in the slightly older Beta Pic moving group.

The inflation of radii by magnetic activity has the capacity to resolve a number of astrophysical problems for low mass PMS stars, including: discrepancies between measured masses, radii, luminosities and temperatures of PMS binary systems; discrepancies between the ages determined from the HRD/CMD for low-mass PMS stars and their higher mass siblings; and the patterns and dispersion of Li depletion seen among low-mass PMS and ZAMS stars. This scenario would require an increase in the timescales of PMS evolution of at least a factor of two, and since observational constraints on timescales for the evolution of circumstellar disks and planetary formation are keyed in to these PMS timescales, they would have to be revised upwards by a similar factor. In addition, the estimation of stellar masses from positions in the CMD/HRD would be significantly affected. PMS stars that are inflated by magnetic activity would have masses that are larger than implied by standard evolutionary models.

\section{ACKNOWLEDGEMENTS}

RDJ and RJJ acknowledge support from the UK Science and Technology Facilities Council (STFC). Based on data products from observations made with ESO Telescopes at the La Silla Paranal Observatory under programme ID 188.B-3002. These data products have been processed by the Cambridge Astronomy Survey Unit (CASU) at the Institute of Astronomy, University of Cambridge, and by the FLAMES/UVES reduction team at INAF/Osservatorio Astrofisico di Arcetri. These data have been obtained from the Gaia-ESO Survey Data Archive, prepared and hosted by the Wide Field Astronomy Unit, Institute for Astronomy, University of Edinburgh, which is funded by the STFC. This publication makes use of data products from the Two Micron All Sky Survey, which is a joint project of the University of Massachusetts and the Infrared Processing and Analysis Center/California Institute of Technology, funded by the National Aeronautics and Space Administration and the National Science Foundation.

This work was partly supported by the European Union FP7 programme through ERC grant number 320360 and by the Leverhulme Trust through grant RPG-2012-541. We acknowledge the support from INAF and Ministero dell' Istruzione, dell' Università' e della Ricerca (MIUR) in the form of the grant "Premiale VLT 2012". This research was partially supported by INAF through a PRIN-2014 grant. The results presented here benefit from discussions held during the Gaia-ESO workshops and conferences supported by the ESF (European Science Foundation) through the GREAT Research Network Programme.

\section{REFERENCES}

Allard F., 2014, in Booth M., Matthews B. C., Graham J. R., eds, Exploring the Formation and Evolution of Planetary Systems Vol. 299 of IAU Symposium, The BT-Settl Model Atmospheres for Stars, Brown Dwarfs and Planets. pp 271-272

Azulay R., Guirado J. C., Marcaide J. M., Martí-Vidal I., Ros E., Jauncey D. L., Lestrade J.-F., Preston R. A., Reynolds J. E., Tognelli E., Ventura P., 2015, , 578, A16

Baraffe I., Homeier D., Allard F., Chabrier G., 2015, , 577, A42

Barrado D., Bouy H., Bouvier J., Moraux E., Sarro L. M., Bertin E., Cuillandre J. C., Stauffer J. R., Lillo-Box J., Pollock A., 2016, ArXiv e-prints, 1608.02751

Bell C. P. M., Naylor T., Mayne N. J., Jeffries R. D., Littlefair S. P., 2013, , 434, 806

Bildsten L., Brown E. F., Matzner C. D., Ushomirsky G., 1997, , 482, 442

Binks A. S., Jeffries R. D., 2016, , 455, 3345

Bouvier J., Lanzafame A. C., Venuti L., et al. 2016, , 590, A78

Boyajian T. S., von Braun K., van Belle G., et al. 2012, , 757, 112

Carpenter J. M., 2001, , 121, 2851

Chen Y., Girardi L., Bressan A., Marigo P., Barbieri M., Kong X., 2014, , 444, 2525

Covey K. R., Agüeros M. A., Law N. M., Liu J., Ahmadi A., Laher R., Levitan D., Sesar B., Surace J., 2016, , 822, 81

David T. J., Hillenbrand L. A., Cody A. M., Carpenter J. M., Howard A. W., 2016, , 816, 21

Demory B.-O., Ségransan D., Forveille T., Queloz D., Beuzit J.L., Delfosse X., di Folco E., Kervella P., Le Bouquin J.-B., Perrier C., Benisty M., Duvert G., Hofmann K.-H., Lopez B., Petrov R., 2009, , 505, 205

Dotter A., Chaboyer B., Jevremović D., Kostov V., Baron E., Ferguson J. W., 2008, , 178, 89

Eldridge J. J., 2009, , 400, L20

Feiden G. A., 2016, ArXiv e-prints, 1604.08036

Feiden G. A., Chaboyer B., 2013, , 779, 183

Feiden G. A., Chaboyer B., 2014, , 789, 53

Fischer D. A., Marcy G. W., 1992, , 396, 178

Frasca A., Biazzo K., Lanzafame A. C., et al. 2015, , 575, A4 
Gilmore G., Randich S., Asplund M., Binney J., Bonifacio P., Drew J., Feltzing S., Ferguson A., Jeffries R., Micela G., et al. 2012, The Messenger, 147, 25

Hillenbrand L. A., White R. J., 2004, , 604, 741

Jackson R. J., Jeffries R. D., 2014a, , 445, 4306

Jackson R. J., Jeffries R. D., 2014b, , 441, 2111

Jackson R. J., Jeffries R. D., Maxted P. F. L., 2009, , 399, L89

Jackson R. J., Jeffries R. D., Randich S., Bragaglia A., Carraro G., Costado M. T., Flaccomio E., Lanzafame A. C., Lardo C., Monaco L., Morbidelli L., Smiljanic R., Zaggia S., 2016, , 586, A52

Jeffries R. D., Jackson R. J., Cottaar M., et al. 2014, , 563, A94

Jeffries R. D., Naylor T., Walter F. M., Pozzo M. P., Devey C. R., 2009, , 393, 538

Kraus A. L., Cody A. M., Covey K. R., Rizzuto A. C., Mann A., Ireland M., Jensen E. L. N., Muirhead P. S., 2016, in American Astronomical Society Meeting Abstracts Vol. 227 of American Astronomical Society Meeting Abstracts, The Mass-Radius Relation of Young Stars from K2. p. 236.12

Kraus A. L., Cody A. M., Covey K. R., Rizzuto A. C., Mann A. W., Ireland M. J., 2015, , 807, 3

López-Morales M., Ribas I., 2005, , 631, 1120

MacDonald J., Mullan D. J., 2013, , 765, 126

Malo L., Doyon R., Feiden G. A., Albert L., Lafrenière D., Artigau É., Gagné J., Riedel A., 2014, , 792, 37

Messina S., Lanzafame A. C., Feiden G. A., Millward M., Desidera S., Buccino A., Curtis I., Jofre' E., Kehusmaa P., Medhi B. J., Monard B., Petrucci R., 2016, ArXiv 1607.06634

Morales J. C., Ribas I., Jordi C., 2008, , 478, 507

Morales J. C., Ribas I., Jordi C., Torres G., Gallardo J., Guinan E. F., Charbonneau D., Wolf M., Latham D. W., Anglada-Escudé G., Bradstreet D. H., Everett M. E., O’Donovan F. T., Mandushev G., Mathieu R. D., 2009, , 691, 1400

Morales J. C., Torres G., Marschall L. A., Brehm W., 2009, , 707, 671

Mullan D. J., MacDonald J., 2001, , 559, 353

Naylor T., 2009, , 399, 432

O’Neal D., Neff J. E., Saar S. H., Cuntz M., 2004, , 128, 1802

Palla F., Randich S., Flaccomio E., Pallavicini R., 2005, Astrophys. J. Letters, 626, L49

Pecaut M. J., Mamajek E. E., Bubar E. J., 2012, , 746, 154

Pozzo M., Jeffries R. D., Naylor T., Totten E. J., Harmer S., Kenyon M., 2000, , 313, L23

Prisinzano L., Damiani F., Micela G., et al. 2016, , 589, A70

Randich S., Gilmore G., Gaia-ESO Consortium 2013, The Messenger, 154, 47

Rizzuto A. C., Ireland M. J., Dupuy T. J., Kraus A. L., 2016, , 817, 164

Sandberg Lacy C. H., Fekel F. C., Pavlovski K., Torres G., Muterspaugh M. W., 2016, , 152, 2

Skrutskie M. F., Cutri R. M., Stiening R., et al. 2006, , 131, 1163

Soderblom D. R., Hillenbrand L. A., Jeffries R. D., Mamajek

E. E., Naylor T., 2014, Protostars and Planets VI, pp 219-241

Soderblom D. R., Jones B. F., Balachandran S., Stauffer J. R., Duncan D. K., Fedele S. B., Hudon J. D., 1993, , 106, 1059

Somers G., Pinsonneault M. H., 2014, , 790, 72

Somers G., Pinsonneault M. H., 2015a, , 807, 174

Somers G., Pinsonneault M. H., 2015b, , 449, 4131

Spina L., Randich S., Palla F., et al. 2014, , 567, A55

Stauffer J. R., Jones B. F., Backman D., Hartmann L. W., Barrado y Navascués D., Pinsonneault M. H., Terndrup D. M., Muench A. A., 2003, , 126, 833
Torres G., 2013, Astronomische Nachrichten, 334, 4

Yee J. C., Jensen E. L. N., 2010, , 711, 303

Zapatero Osorio M. R., Béjar V. J. S., Pavlenko Y., Rebolo R., Allende Prieto C., Martín E. L., García López R. J., 2002, , 384, 937

\section{APPENDIX A: THE $V-K$ COLOUR}

The main plots and conclusions of the paper have been checked using the $V-K$ colour in addition to $V-I$. The equivalents to Figs. 1 and 3a,c are shown in Fig. A1. The $K$ magnitudes here are on the 2MASS system (from Skrutskie et al. 2006) and the model $V-K$ colours on the CIT system were transformed to the 2MASS system by subtracting 0.024 mag (Carpenter 2001). The isochrones shown are not fitted but placed at the same ages that are discussed in the main text for the $V-I$ isochrones. The reader can readily see that the problem outlined in Section 2 is also apparent when using $V-K$; Figs. A1a,b show that an age of $7.5 \mathrm{Myr}$ fits the CMD (though a slightly younger age might be better), but the same (or any older) isochrone in the EWLi vs $V-K$ does not match the data. Applying the same 10 per cent level of inflation to the BHAC15 and Dartmouth models at a given mass yields the solid and dotted isochrones plotted in Figs. A1c,d which provide an acceptable fit to both diagrams at an age of $\sim 20 \mathrm{Myr}$. The magnetic Dartmouth models yield an inferior fit at this age, being too blue at lower masses, but qualitatively address the problem in the same way.

\section{APPENDIX B: THE EFFECT OF STARSPOTS ON MAGNITUDE, COLOUR AND LITHIUM EQUIVALENT WIDTH}

A simple two-temperature model is used to determine the effect of starspots on the observable properties of spotted stars (see Jackson \& Jeffries 2014b). Starspots affect the magnitude and colour of stars on the Hayashi track in two ways;

- There is a small increase in the temperature of the unspotted photosphere of the spotted star, $T_{s}$ relative to that of an unspotted star, $T_{u}$ of the same mass and age. From Eqs. (1) \& (2)

$\left(T_{s} / T_{u}\right)_{M, t}=(1-\beta)^{-(3 D-1) / 4}$.

In practice $D \sim 1 / 3$, so $T_{s}$ is only slightly larger than $T_{u}$.

- There is also a contribution to the observed stellar flux from the spotted area of the photosphere that always makes the star appear redder. The relative magnitude of this contribution depends on the spot temperature $T_{s p}$ and the fraction of the surface covered by starspots, $\gamma=\beta /\left(1-T_{s p}^{4} / T_{s}^{4}\right)$

The $V$-band flux is the sum of the flux from the unspotted surface of area $(1-\gamma) 4 \pi R^{2}$ and temperature $T_{s}$, and the flux from the spotted surface of area $\gamma 4 \pi R^{2}$ and temperature $T_{s p}$; giving a combined $V$ band bolometric correction of;

$\mathrm{BC}_{V, \text { star }}=2.5 \log \left(\frac{1-\gamma}{1-\beta} 10^{\frac{\mathrm{BC}_{V, T_{s}}}{2.5}}+\frac{\gamma\left(\frac{T_{s p}}{T_{s}}\right)^{4}}{1-\beta} 10^{\frac{\mathrm{BC}_{V, T_{s p}}}{2.5}}\right)$,

where $\mathrm{BC}_{V, T}$ is the bolometric correction at temperature $T$ given by the BHAC15 model at the appropriate stellar age. A similar expression is used to evaluate the $I$ - and $K$-band bolometric correc- 

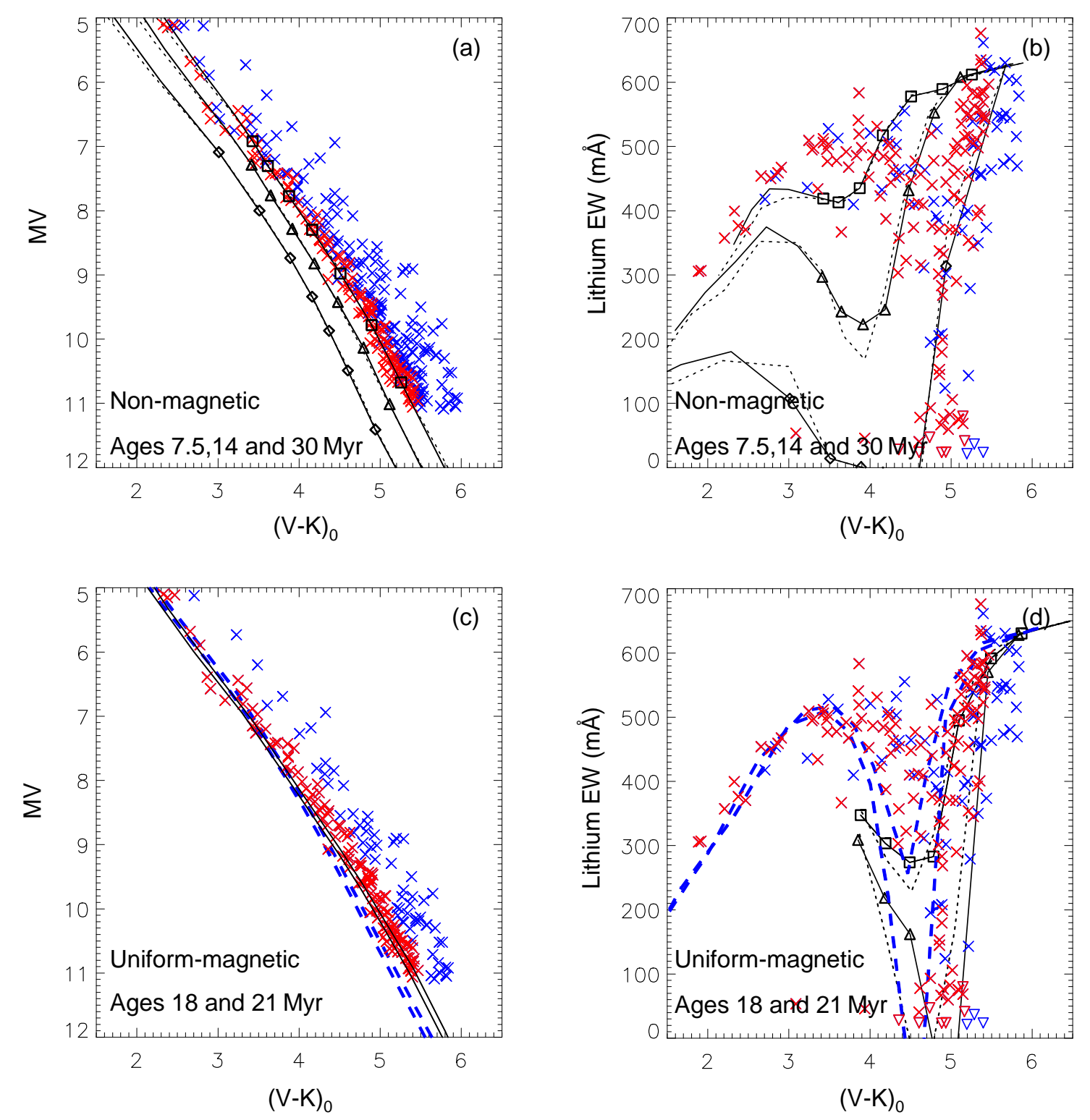

Figure A1. The absolute magnitude versus intrinsic $V-K$ diagram and lithium depletion pattern in the Gamma Vel cluster (same data and symbol colour meaning as for Fig. 1) compared with isochrones calculated using the $V-K$ (2MASS) colour and reddening $E(V-K)=0.113$. Plots (a) and (b) are similar to Fig. 1. Black solid lines and black short-dashed lines are BHAC15 and Dartmouth isochrones respectively at 7.5, 14 and 30 Myr. Plots (c) and (d) show 18 and 21 Myr BHAC15 and Dartmouth isochrones (solid and dotted) modified for 10 per cent radius inflation according to the polytropic model described in Section 3, assuming a uniform surface temperature. The blue dashed lines show the same isochrones for the magnetic Dartmouth model.

tions in order to determine the $V-I$ or $V-K$ colours of the spotted star.

EWLi is also calculated from the curves of growth discussed in Section 2, assuming that both spotted and unspotted areas have the same Li abundance, and that the EWLi contribution, corresponding to the assumed temperatures of the spotted and unspotted regions, is weighted as $\gamma T_{s p}^{4}$ and $(1-\gamma) T_{s}^{4}$ respectively. This in turn assumes that the continuum flux at $6708 \AA$ scales roughly as the bolometric flux. The exact weighting makes little difference since the EWLi vs $T$ relation for a given abundance is quite flat.

This paper has been typeset from a $\mathrm{T}_{\mathrm{E}} \mathrm{X} / \mathrm{L}_{\mathrm{E}} \mathrm{X}$ file prepared by the author. 